\title{
Differentially Expressed Drl and Drl-2 Play Opposing Roles in Wnt5 Signaling during Drosophila Olfactory System Development
}

\author{
Masao Sakurai, ${ }^{1}$ Tomoko Aoki, ${ }^{1}$ Shingo Yoshikawa, ${ }^{2}$ Linda A. Santschi, ${ }^{2,3}$ Hiroko Saito, ${ }^{1}$ Keita Endo, ${ }^{1,4}$ \\ Kyoko Ishikawa, ${ }^{1}$ Ken-ichi Kimura, ${ }^{5}$ Kei Ito, ${ }^{4}$ John B. Thomas, ${ }^{2}$ and Chihiro Hama ${ }^{1}$ \\ ${ }^{1}$ Laboratory for Neural Network Development, RIKEN Center for Developmental Biology, Hyogo 650-0047, Japan, ${ }^{2}$ Molecular Neurobiology Laboratory, \\ Salk Institute for Biological Studies, La Jolla, California 92037-1099, ${ }^{3}$ Coastal Marine Biolabs, Ventura, California 93001, ${ }^{4}$ Institute of Molecular and Cellular \\ Biosciences, University of Tokyo, Tokyo 113-0032, Japan, and ${ }^{5}$ Hokkaido University of Education, Iwamizawa Campus, Hokkaido 068-8642, Japan
}

In Drosophila, odor information received by olfactory receptor neurons (ORNs) is processed by glomeruli, which are organized in a stereotypic manner in the antennal lobe $(\mathrm{AL})$. This glomerular organization is regulated by Wnt 5 signaling. In the embryonic CNS, Wnt 5 signaling is transduced by the Drl receptor, a member of the Ryk family. During development of the olfactory system, however, it is antagonized by Drl. Here, we identify Drl-2 as a receptor mediating Wnt 5 signaling. Drl is found in the neurites of brain cells in the AL and specific glia, whereas Drl-2 is predominantly found in subsets of growing ORN axons. A drl-2 mutation produces only mild deficits in glomerular patterning, but when it is combined with a $d r l$ mutation, the phenotype is exacerbated and more closely resembles the Wnt5 phenotype. Wnt5 overexpression in ORNs induces aberrant glomeruli positioning. This phenotype is ameliorated in the $d r l-2$ mutant background, indicating that Drl-2 mediates Wnt5 signaling. In contrast, forced expression of Drl-2 in the glia of $d r l$ mutants rescues the glomerular phenotype caused by the loss of antagonistic Drl function. Therefore, Drl-2 can also antagonize Wnt5 signaling. Additionally, our genetic data suggest that Drl localized to developing glomeruli mediates Wnt5 signaling. Thus, these two members of the Ryk family are capable of carrying out a similar molecular function, but they can play opposing roles in Wnt 5 signaling, depending on the type of cells in which they are expressed. These molecules work cooperatively to establish the olfactory circuitry in Drosophila.

\section{Introduction}

In the Drosophila olfactory system, odorants are detected by 1300 olfactory receptor neurons (ORNs) located on the antennae and maxillary palps (Stocker, 1994). Each ORN expresses only one or two of the $\sim 60$ odorant receptors (ORs), and ORN axons expressing a given OR converge onto one of the $\sim 50$ glomeruli in the antennal lobe (AL) (Luo and Flanagan, 2007) (see Fig. 1). This wiring configuration represents odor information as a combination of activated glomeruli in the AL. Glomeruli are specialized discrete structures in which ORNs, projection neurons (PNs), and local interneurons specifically form synapses (see Fig. 1A). PNs extend dendrites into the AL and extend axons into higher brain centers, including the mushroom body and protocerebrum (Stocker, 1994). These neurons are generated during larval stages

\footnotetext{
Received June 19, 2008; revised Jan. 22, 2009; accepted Feb. 21, 2009.

This work was supported in part by a research grant from the Brain Science Foundation (Japan) and by a Grantin-Aid for Scientific Research (B) from the Japan Society for the Promotion of Science. We thank L. Luo, L. Vosshall, B. Dickson, T. Aigaki, H. Hing, N. K. Tanaka, National Institute of Genetics, Mitsubishi Kagaku Institute of Life Sciences, the Bloomington Stock Center and the Drosophila Genetic Resource Center, and Kyoto Institute of Technology for fly stocks, and L. Fradkin and Developmental Studies Hybridoma Bank for antibodies. We are grateful to S. Hayashi and F. Matsuzaki for the use of facilities and equipment. We also thank $\mathrm{H}$. Hing for communicating data before publication, and all members of the Hama Laboratory and Center for Developmental Biology for helpful discussion.

Correspondence should be addressed to Chihiro Hama, RIKEN Center for Developmental Biology, 2-2-3 Minatojima-minami, Chuo, Kobe, Hyogo 650-0047, Japan. E-mail: hama@cdb.riken.jp.

D0I:10.1523/JNEUROSCI.2821-08.2009

Copyright $\odot 2009$ Society for Neuroscience $\quad$ 0270-6474/09/294972-09\$15.00/0
}

from three neuroblasts (NBs) arranged around the developing AL. Previous studies demonstrated that ORNs and PNs use independent mechanisms to organize neurite projections that precede the emergence of the stereotyped glomerular organization (Jefferis et al., 2004; Komiyama et al., 2004). Several molecules involved in transcriptional regulation (Komiyama et al., 2003, 2004; Endo et al., 2007) and axon guidance (Jhaveri et al., 2004; Komiyama et al., 2007; Lattemann et al., 2007; Sweeney et al., 2007) function in ORNs or PNs to establish glomerular patterning.

Wnt5 signaling controls the development of the olfactory system (Yao et al., 2007). Wnt5 is a guidance cue that repels a subset of commissural axons along the anterior-posterior axis in the CNS of Drosophila embryos (Yoshikawa et al., 2003). The repulsive Wnt5 signal from the posterior commissure is transduced by the Drl receptor, which is localized to the growth cones and axons of neurons that project through the anterior commissures (Bonkowsky et al., 1999). Drl is a member of the Ryk subfamily of atypical receptor tyrosine kinases. In vertebrates, Wnt-Ryk signaling repels axons in several neural tissues (Liu et al., 2005; Keeble et al., 2006; Schmitt et al., 2006). This signaling is also required for axonal growth in the Drosophila mushroom body (Grillenzoni et al., 2007) and induces neurite outgrowth in vertebrates (Lu et al., 2004). During development of the Drosophila olfactory system, Wnt5 produced by ORNs (supplemental Fig. 1, available at www.jneurosci.org as supplemental material) regu- 

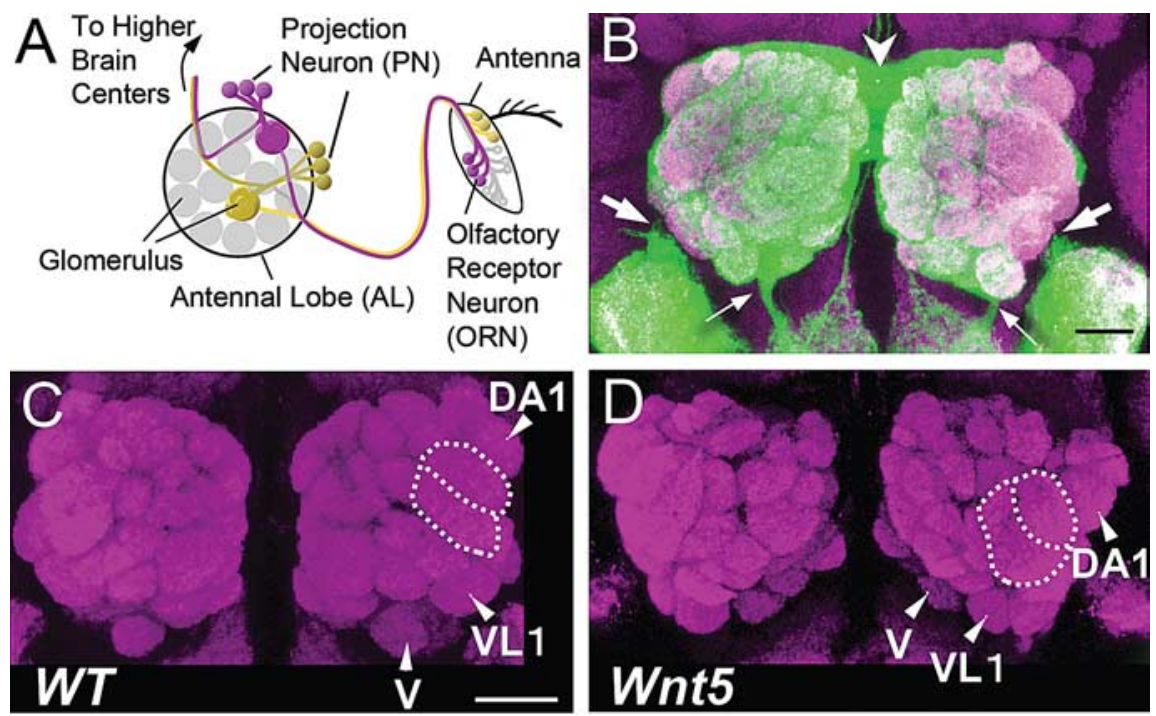

Figure 1. A, Schematic of the Drosophila olfactory system. See text for the details. $\boldsymbol{B}$, Structure of the AL. The thick and thin arrows indicate the entry sites of ORN axons into the ALs from the antennae and maxillary palps, respectively. Two ALs are connected by the commissural bundle of ORN axons (arrowhead). ORNs and other neural cells are stained with mCD8::GFP (green) driven by pebbled-Gal4. C, D, The distribution of glomeruli in the wild-type $(\boldsymbol{C})$ and Wnt5 mutant $(\boldsymbol{D})$ ALs. Compare the positions of three glomeruli DA1, VL1, and V. The dotted lines delineate VA1d (dorsal) and VA1v (ventral). Brains are stained with nc 82 that labels presynaptic regions (magenta). Dorsal is up. Scale bars, $20 \mu \mathrm{m}$.

lates AL organization, whereas Drl, expressed by numerous brain cells including specific glia, antagonizes Wnt5 signaling (Yao et al., 2007). However, the receptor that mediates Wnt5 signaling has not been identified.

In the current study, we demonstrate that Drl-2 functions as a Wnt5 receptor that mediates Wnt5 signaling and can also antagonize Wnt5 when ectopically expressed in glia. In addition, our genetic data suggest that Drl localized to developing glomeruli functions as a Wnt5 receptor. We propose that Drl and Drl-2, which carry out similar molecular functions, play opposing roles in Wnt5 signaling, depending on the types of cells in which they are expressed during development. These molecules cooperate to establish the olfactory circuitry in Drosophila.

\section{Materials and Methods}

Strains. $W n t 5^{D 7}, d r l^{R 343}$, and $d r l-2^{E 124}$ are the null alleles (Callahan et al., 1995; Yoshikawa et al., 2003; Inaki et al., 2007) that were used throughout this study. To drive gene expression in ORNs, we used enhancer trap Gal4 lines, AM29 (Endo et al., 2007), lz-Gal4 (Jhaveri et al., 2004), SG18.1 (Jhaveri and Rodrigues, 2002), and pebbled-Gal4 (Sweeney et al., 2007), as well as the following marker lines expressing an odorant receptor (Or) promoter fusion: Or-Gal4: Or22a, Or23a, Or46a, Or47a, Or47b (Vosshall et al., 1999), Or42a, Or83c, Or85e, Or92a (Komiyama et al., 2004), Or43a, Or71a (Fishilevich and Vosshall, 2005), and Gr21a (Scott et al., 2001); and Or-mCD8::GFP: Or9a, Or10a, Or43b, Or85a, and Or85d (Couto et al., 2005). To label both the DM6 and DL4 glomeruli simultaneously, either AM29 or $l z$-Gal4 was used. To drive gene expression in PNs, the enhancer trap Gal4 lines GH146 (Stocker et al., 1997) ( $\sim 90$ of 150 PNs), NP21 (Kimura et al., 2005) (DA1, VA1d,v), NP5103 (Tanaka et al., 2004) (VM2), and NP7247 (VL1) were used in this study. GS1192 (Toba et al., 1999) carries a UAS insertion in the $5^{\prime}$ region of the Wnt5 gene and was used to express Wnt5 in ORNs after a cross with AM6.

Clonal analysis. To generate $\mathrm{drl}$ clones in second-order neurons or $d r l-2$ clones in ORNs, the mosaic analysis with a repressible cell marker (MARCM) method was used as described previously (Lee and Luo, 1999). To induce $d r l$ and $d r l-2$ clones by $h s F L P$, larvae were heat-shocked for $1.5 \mathrm{~h}$ at $37^{\circ} \mathrm{C}$ during the first- and second-instar stages, respectively. The genotypes used were as follows: $y w h s F L / w$; $d r l^{R 343}$ FRT40A Gal4-GH146/tubP-Gal80 FRT40A; UAS-mCD8::GFP, and $y w h s F L P / w ; F R T G 13 \mathrm{drl}-2^{E 124} / F R T G 13$ tubP-Gal80; Or59c-Gal4/UAS-mCD8::GFP. The following genotypes were used to generate large $d r l-2$ clones in ORNs: $y w$ eyFLP/w; FRTG13 drl-2 $2^{E 124} /$ FRTG13 tubP-Gal80; Or59cGal4/UAS-mCD8::GFP.

Immunohistochemistry. To stain the ALs, brains were dissected from adult flies or pupae and fixed with $4 \%$ paraformaldehyde for $60-90$ min on ice. The following primary antibodies were used in this study: mouse monoclonal nc82 (1:10) (Wagh et al., 2006), rat anti$\operatorname{mCD} 8 \alpha \quad(1: 100 ;$ Caltag), rabbit anti-GFP (1:500; MBL), rat anti-Drl (1:500) (Bonkowsky et al., 1999), and guinea pig anti-Drl-2 (1:1000).

Wnt5 staining. Dissected brains were incubated with anti-Wnt5 (1:50) (Fradkin et al., 1995 ) in PBS for $30 \mathrm{~min}$ at room temperature, washed twice for $5 \mathrm{~min}$ in PBS, and fixed with PLP (paraformaldehyde/lysine/periodate) fixative for $20 \mathrm{~min}$ at room temperature (Yao et al., 2007).

Antibody generation. An Drl-2 antibody was generated by immunizing a guinea pig against a polypeptide containing the $207-380$ aa sequence of the cytoplasmic region of Drl-2. The corresponding DNA fragment was cloned into a His-tag expression vector, and the fused polypeptide was purified and injected into guinea pigs. The antiserum was purified through an affinity column.

Construction of transgenic flies. To generate transgenic fly lines carrying $U A S-d r l-2$, a full length of $d r l-2$ cDNA was cloned into the pUAST transformation vector and injected into fly embryos for germline transformation according to a standard protocol.

\section{Results}

\section{AL defects in Wnt5 and $d r l$ mutants}

In the wild-type fly brain, ORN axons extending from the antennae and maxillary palps project to the ipsilateral AL. Many axons also project to the contralateral AL, forming a commissure between the two ALs (Fig. 1 B). In Wnt5 mutants, this commissure is frequently lost as previously reported (Yao et al., 2007) (supplemental Fig. $2 B$, available at www.jneurosci.org as supplemental material), and the ALs resemble inverted triangles (Fig. 1D), in contrast to the globular shape of wild-type ALs (Fig. 1C). To characterize this mutant phenotype in greater detail, we specifically labeled 12 glomeruli (Fig. $2 A, B$; supplemental Fig. 3, available at www.jneurosci.org as supplemental material) with enhancer-trapped Gal4 and OR-Gal4 lines. In Wnt5 mutants, many of the glomeruli located in the periphery of the AL were rotated in a clockwise manner in the right AL (Fig. 2C). Although the glomeruli in the ALs without a commissure tended to rotate more than those with a commissure, the phenotype was similar for both cases (supplemental Fig. 2, available at www.jneurosci. org as supplemental material). Because Wnt5 signaling is transduced through Drl in the embryonic CNS, we next examined the $\mathrm{AL}$ phenotype of $d r l$ mutants. In the $d r l$ mutant brain, the commissure was frequently lost $(50 \% ; n=14)$ (Fig. $3 B)$ as observed in Wnt5 mutants, but the overall shape of the ALs, as well as the glomerular distribution, differed from that in the Wnt5 AL (Fig. $2 A, D$; supplemental Fig. 3, available at www.jneurosci.org as supplemental material). Three glomeruli located in the medial $\mathrm{AL}$ region of $d r l$ mutants were shifted in the same direction observed in Wnt5 (Fig. 2 D, blue arrows), but several lateral glomeruli were relocated in a pattern distinct from the Wnt5 pattern (Fig. 2D, orange arrows). The most conspicuous differences be- 

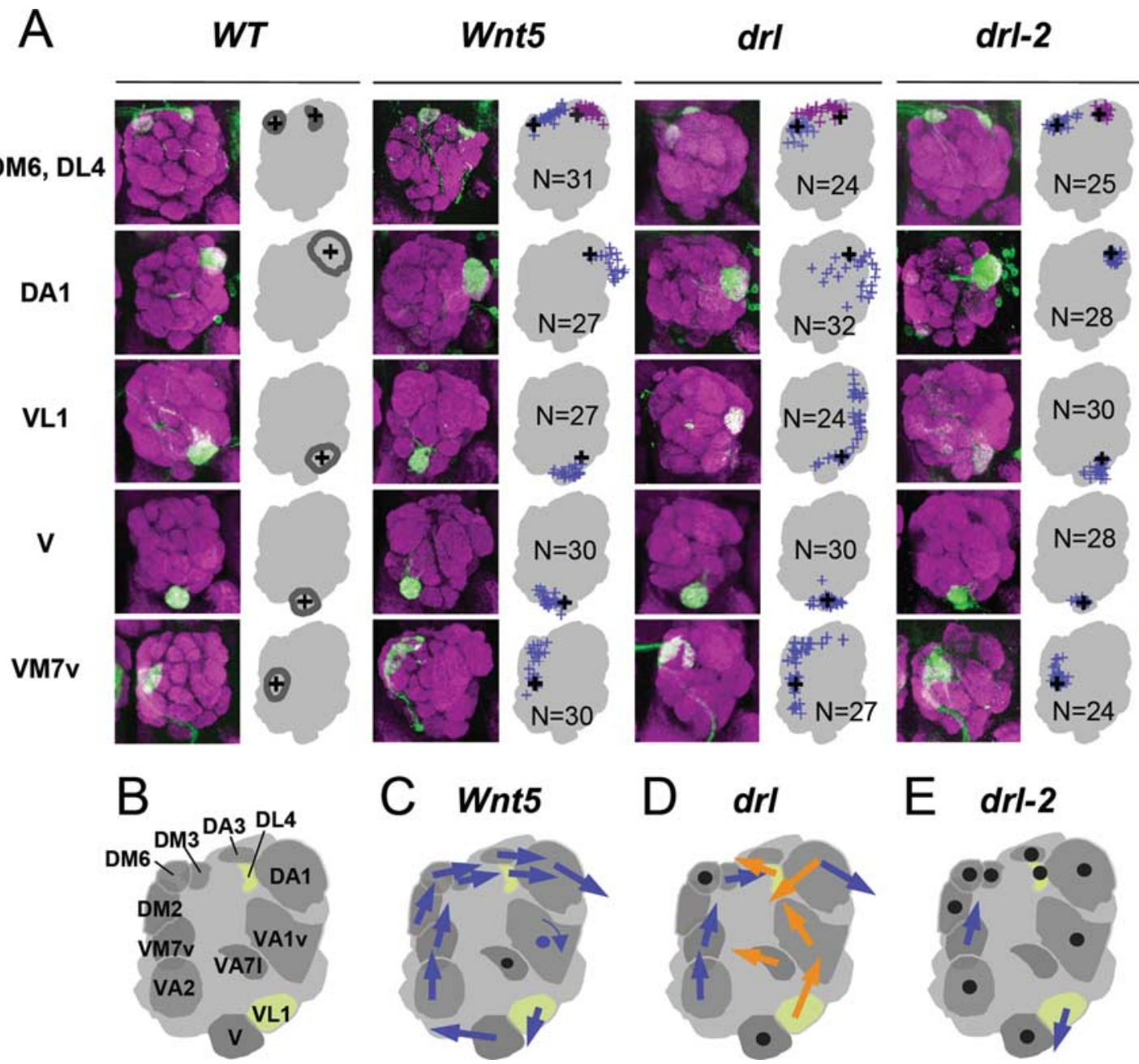

\section{drl drl-2}
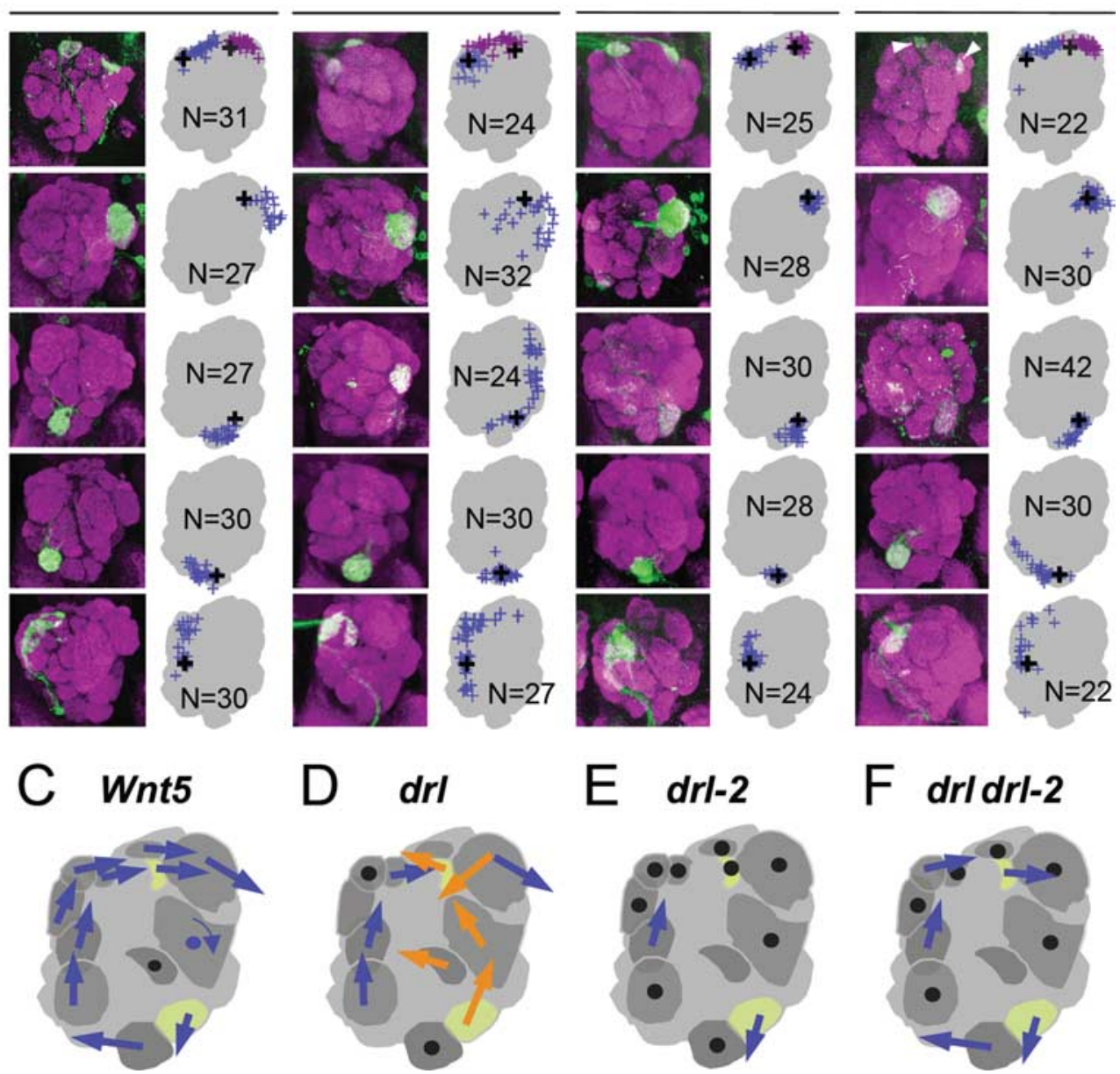

Figure 2. The distribution of glomeruli in Wnt5 and other mutants. A, The positions of six glomeruli DM6, DL4, DA1, VL1, V, and VM7v in Wnt5, drl, drl-2, and drl drl-2 mutants. For analysis of the other glomeruli, see supplemental Figures 3 and 4 (available at www.jneurosci.org as supplemental material). N indicates the number of ALs examined. The black plus signs represent the positions of glomeruli in the wild-type ALs. The blue and magenta (DL4) plus signs indicate the positions of glomeruli in the mutants. B, Distribution of the 12 glomeruli examined in this study. $C-F$, Summary of movement of glomeruli in $W n t 5(\boldsymbol{C}), d r l(\boldsymbol{D}), d r l-2(\boldsymbol{E})$, and $d r l d r l-2(\boldsymbol{F})$ mutants. The blue and orange arrows represent the shift of individual glomeruli in the same direction as in $W n t 5$ mutants or in a different direction, respectively. Glomeruli that retained their original positions are shown by black dots. VA1v in the Wnt5 AL appears to rotate in the direction indicated by the thin arrow. DL4 and VL1 are labeled with yellow in $\boldsymbol{B}-\boldsymbol{F}$. Lateral is to the right.

tween $d r l$ and Wnt5 mutants were the locations of VL1 and DL4 glomeruli (Fig. $2 B-F$, yellow); these glomeruli moved clockwise in Wnt5 mutants, but they shifted counterclockwise in $d r l \mathrm{mu}-$ tants (Fig. 2, compare $D, C$ ). Thus, the $d r l$ AL exhibits a complex glomerular pattern that partially overlaps with the glomerular pattern in the Wnt5 AL.

Drl that is expressed in specific glial processes outside of the AL during pupal stages regulates AL development by antagonizing Wnt5 signaling (Yao et al., 2007). In addition to the glia, Drl is also present in the developing ALs (Fig. 4). In pupae of $21 \mathrm{~h}$ after puparium formation (APF), immunostaining for Drl was detectable in discrete regions of the anterior $\mathrm{AL}$ with stronger staining in the lateral-ventral portion (Fig. $4 A$ ). In the posterior AL, Drl was detected only in the dorsal-lateral area (Fig. $4 B$ ). This staining pattern persisted at $42 \mathrm{~h} \mathrm{APF} \mathrm{(Fig.} 4 D$ ). In the anterior AL of $42 \mathrm{~h}$ APF pupae, Drl was found in several developing glomeruli at varying levels of intensity (Fig. $4 C$ ). One of these glomeruli was DA1, which was labeled with Gal4-NP21 (Fig. 4E, E'). Thus, Drl is distributed in a characteristic pattern in the AL during these developmental stages.

To investigate the function of $\mathrm{Drl}$ in the $\mathrm{AL}$, we used the
MARCM system to generate $d r l$ mutant clones in lineages of the three NBs that form the AL (Fig. 5). In this experiment, Gal4GH146 (Stocker et al., 1997) was used to drive UAS-mCD8::GFP expression in subsets of cells derived from the three NBs. The overall shape and DA1 glomerular pattern were normal in $\operatorname{DrlNB}$ clones originating from the anterior dorsal neuroblast (adNB) ( $94 \%, n=34$ for mutants, vs $97 \%, n=19$ for wild type) (Fig. $5 A, D)$. In contrast, the ALs containing $d r l \mathrm{NB}$ clones derived from the lateral neuroblast (lNB) displayed an abnormal shape and DA1 was shifted ventrally to the lateral side of VA1d in these ALs (correct DA1 position: $33 \%, n=30$ for mutants, vs $92 \%, n=$ 12 for wild type) (Fig. $5 B, E, H)$. This defect is similar to the phenotype of $W n t 5$ mutants. NB clones derived from the ventral neuroblast $(\mathrm{vNB})$ yielded dendritic projections into DA1 that were often sparse or stunted $(64 \%, n=28$ for mutants, vs $5 \%$, $n=19$ for wild type) (Fig. 5C,F). However, both the overall shape of the AL and the projections into most glomeruli were indistinguishable from wild type. Therefore, Drl functions in the progeny of $1 \mathrm{NB}$ to control DA1 positioning and in the progeny of $\mathrm{vNB}$ to produce the normal dendritic arborization into DA1.

To test whether the DA1 position phenotype in the $\mathrm{INB}$ clones 

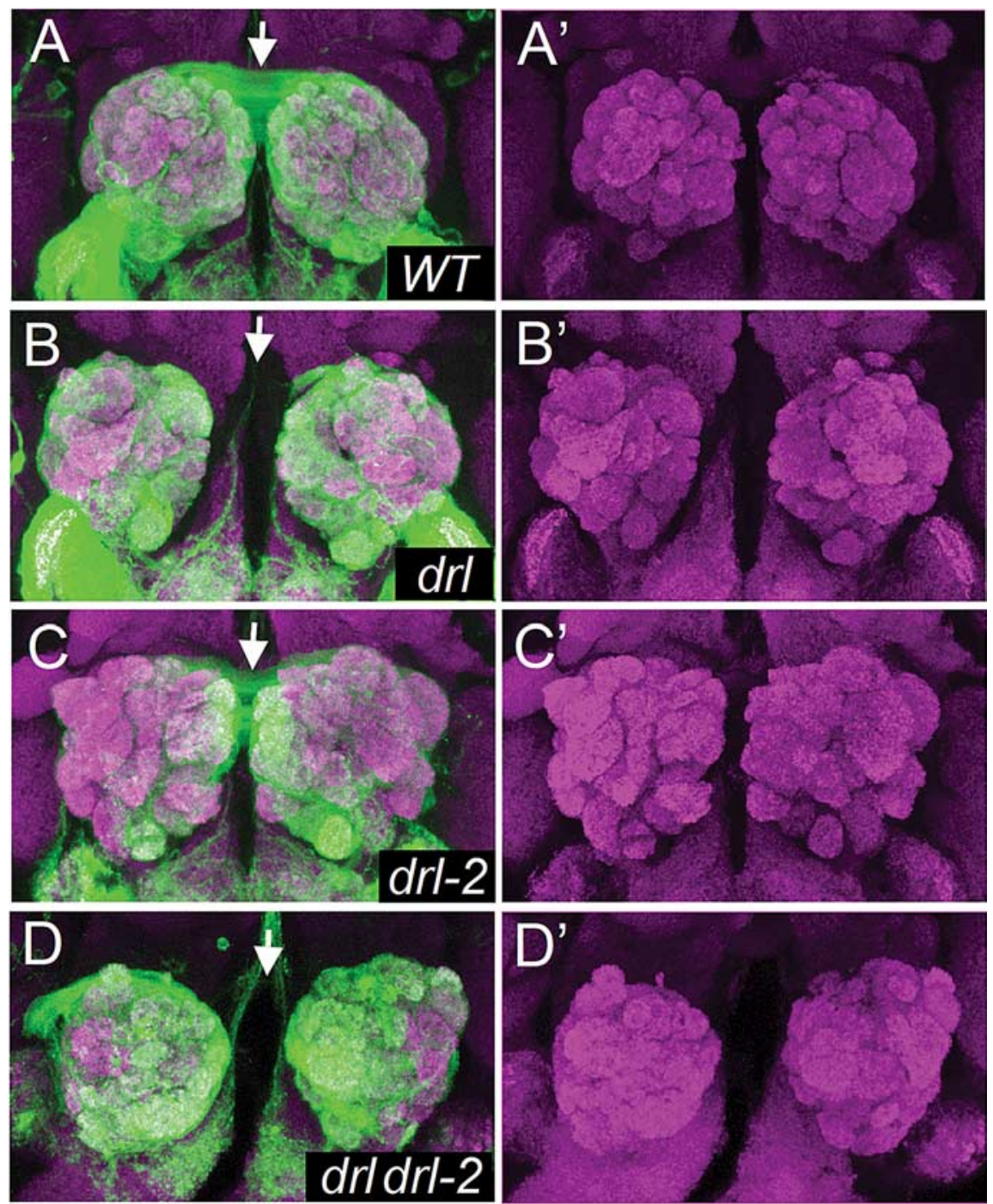

Figure 3. Defects in commissure formation. $\boldsymbol{A}, \boldsymbol{A}^{\prime}$, Wild type. $\boldsymbol{B}, \boldsymbol{B}^{\prime}, d r l . \boldsymbol{C}^{\prime} \boldsymbol{C}^{\prime}, d r l-2 . \boldsymbol{D}, \boldsymbol{D}^{\prime}, d r l d r l-2 . \boldsymbol{A}-\boldsymbol{D}$, ALs are stained for mCD8::GFP (green) to visualize ORN axons and also counterstained with nc82 (magenta). pebbled-Gal4 is used to drive UAS-mCD8::GFP. Note that the commissural axon bundle (arrow) is retained in wild type $(\boldsymbol{A})$ and $d r l-2(\boldsymbol{C})$, but disrupted in $d r l(\boldsymbol{B})$ and $d r l d r l-2$ (D).

is caused by a cell-autonomous function of Drl within $d r l$ expressing cells or by a more complex mechanism, we performed two sets of experiments. First, we examined single-cell clones projecting to DA1, using a specific glomerular marker (Fig. 5G). The dendrites of single $d r l$ cells innervated DA1 normally (100\%; $n=5$ ). Second, we performed a rescue experiment in which UAS-drl expression was driven by Gal4-GH146 in the $d r l$ lNB clones. Drl expression, however, failed to restore the DA1 position phenotype (correct DAl position: $42 \%, n=31$ ) (Fig. $5 H$ ). These results suggest that the correct positioning of DA1 may reflect the cooperation of many INB progeny cells, some of which are not expressing Gal4-GH146. Thus, although it is not yet clear how AL-localized Drl organizes the glomerular pattern, Drl expressed both in the glia outside of the AL and in $\mathrm{vNB}$ and $\mathrm{NNB}$ progenies is essential for regulating Wnt 5 signaling required for proper AL development.

\section{AL defects in drl-2 mutants}

Overlapping, but distinct, glomerular defects in Wnt5 and $d r l$ mutants suggest the presence of other receptors that mediate
Wnt5 signaling. Drl-2 is one candidate receptor because it is similar to Drl; it contains an extracellular WIF (Wnt inhibitory factor) domain and shares 35\% amino acid identity with Drl throughout its length. Drl-2 mutants display mild AL defects, but the commissure between the ALs remains intact (Fig. 3C). We examined the distribution of 11 glomeruli with specific markers. Two glomeruli were observed in aberrant locations (Fig. 2A,E; supplemental Fig. 4, available at www.jneurosci.org as supplemental material). VM7v was shifted dorsally slightly, whereas VL1 was positioned ventrally (Fig. 2E). The direction of VM7v movement was the same in $d r l-2$, $W n t 5$, and $d r l$ mutants (Fig. 2C-E). In contrast, VL1 moved in opposite directions in $d r l-2$ and $d r l$ mutants but in the same direction in $d r l-2$ and Wnt5 mutants (Fig. 2C-E). The similarity between $d r l-2$ and Wnt5 mutants suggests that Drl-2 may contribute to Wnt5 signaling.

The double $d r l$ and $d r l-2$ mutant exhibited a more striking phenotype. The ALs in $d r l d r l-2$ mutants frequently lacked the commissure $(30 \% ; n=13)$ (Fig. 3D), and five glomeruli were repositioned in a clockwise manner similar to the defects in Wnt5 mutants (Fig. $2 A, F$; supplemental Fig. 4, available at www. jneurosci.org as supplemental material). Notably, whereas the DL4 and VL1 glomeruli shifted in opposite directions in $d r l$ and Wnt5 mutants (Fig. 2C,D, yellow), in the $d r l d r l-2$ double mutant they shifted in the same direction as Wnt5 mutants (Fig. 2 , compare $F, C$ ). This result further supports the hypothesis that Drl-2 mediates Wnt5 signaling.

\section{Drl and Drl-2 have different expression patterns}

To determine the expression pattern of Drl-2, we raised an antibody against the Drl-2 protein. This Drl-2 staining was absent in $d r l-2$ mutant brains (Fig. $6 E$ ), indicating that the antibody is specific for Drl-2. At $24 \mathrm{~h} \mathrm{APF}$, when ORN axons have already invaded the developing AL region, Drl-2 was localized to axons navigating along the lateral tract (Fig. $6 \mathrm{~A}$ ). Drl-2 was also present in the region adjacent to the exit site of ORN axons from the dorsal AL (Fig. 6A, arrows). At later stages, an increasing number of ORN axons along both lateral and medial tracts were immunopositive for Drl-2 (Fig. $6 B, D$ ). However, a fraction of ORN axons including those extending from the maxillary palps were not stained. At $42 \mathrm{~h}$ APF, Drl-2 staining was present in several glomeruli and at the commissure (Fig. 6C). These signals disappeared by $72 \mathrm{~h} \mathrm{APF} \mathrm{(data} \mathrm{not}$ shown). In contrast to the predominant ORN axon staining pattern of Drl-2, Drl was localized to the neurites of neurons surrounding the AL in the brain (Fig. 6F) (also see Fig. 4). Thus, during AL development, Drl and Drl-2 are generally expressed in distinct cell types. 

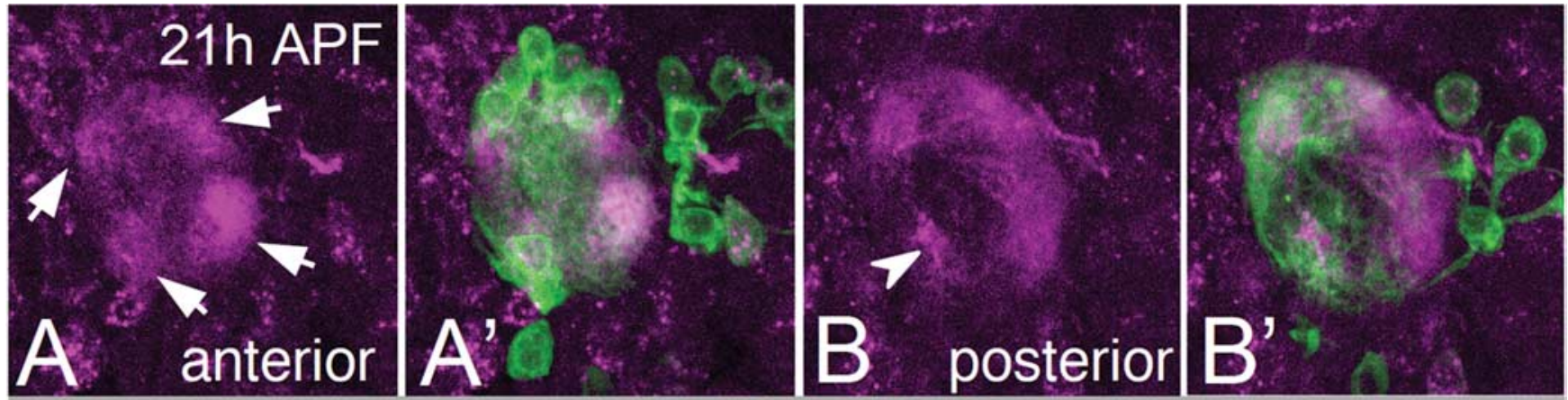

\section{a-Drl GH146}
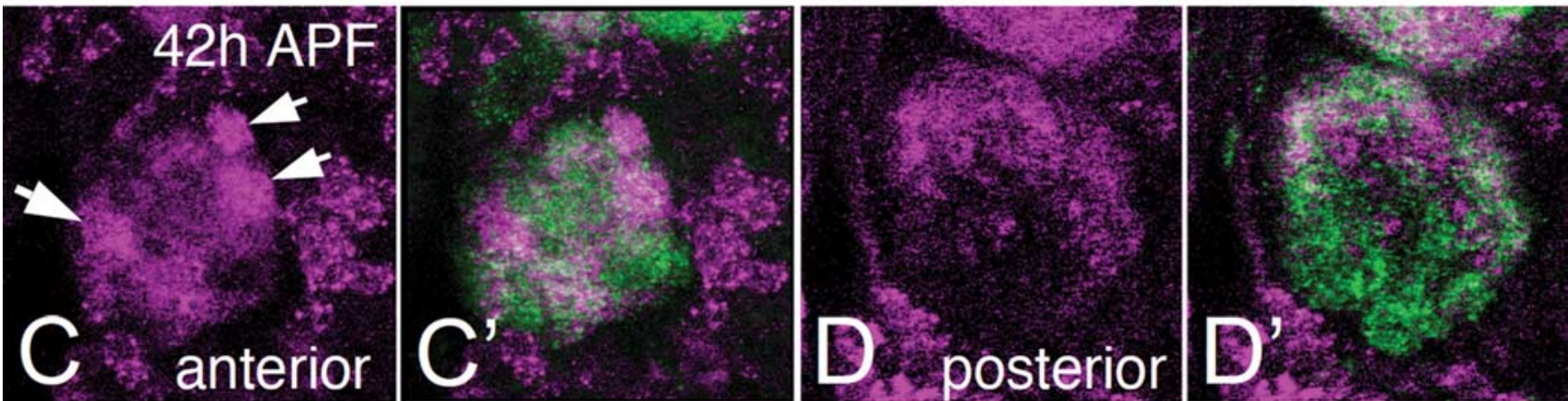

\section{a-DrI nc82}

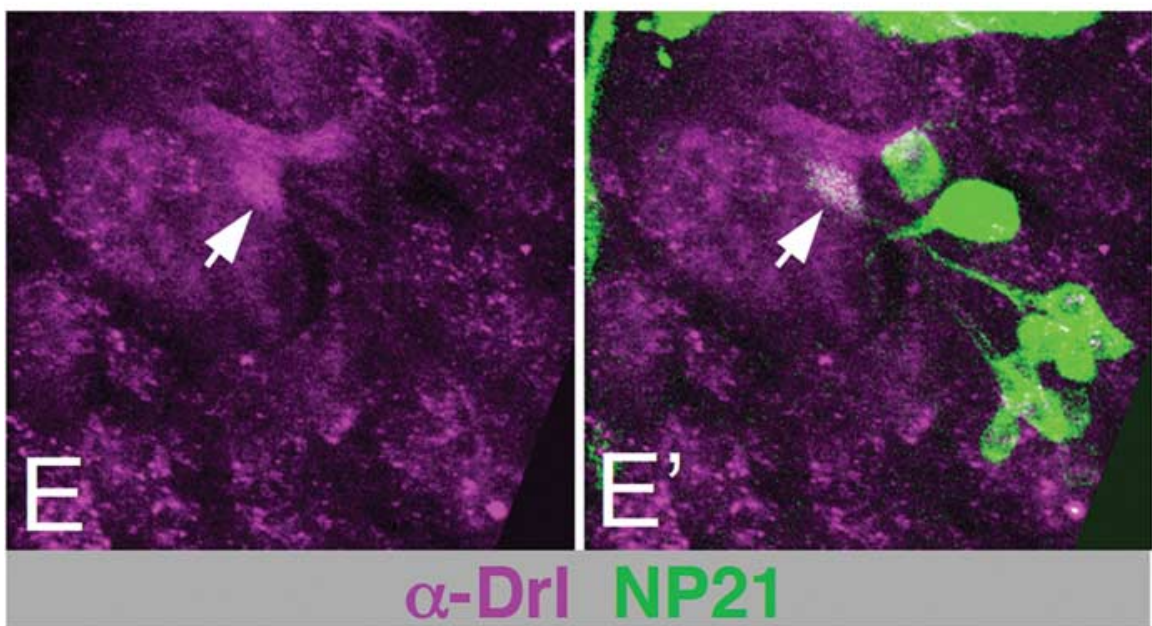

Figure 4. Localization of Drl protein in the ALs. $\boldsymbol{A}-\boldsymbol{B}^{\prime}$, Twenty-one hours APF. $\boldsymbol{A}, \boldsymbol{A}^{\prime}$, Anterior AL. Drl immunostaining (magenta) is prominently observed in four spots (arrows). $\boldsymbol{B}, \boldsymbol{B}^{\prime}$, Posterior AL. Drl is detected in the dorsal-lateral region of the AL. An arrowhead in $\boldsymbol{B}$ indicates the exit site of Drl-positive neurites toward higher brain centers. PNs are labeled with $\mathrm{mCD}$ \%::GFP (green) driven by Gal4-GH146 in $\boldsymbol{A}^{\prime}$ and $\boldsymbol{B}^{\prime}$. C- $\boldsymbol{D}^{\prime}$, Forty-two hours APF. $\boldsymbol{C}, \boldsymbol{C}^{\prime}$, Anterior AL. Drl signals (magenta) are prominent in a few spots (C, arrows). $\boldsymbol{D}, \boldsymbol{D}^{\prime}$, Posterior AL. Drl is detected in the dorsal-lateral region of the AL. The AL is counterstained with nc82 (green) in $\boldsymbol{C}^{\prime}$ and $\boldsymbol{D}^{\prime}$. $\boldsymbol{E}, \boldsymbol{E}^{\prime}$, Drl expression in DA1, $33 \mathrm{~h}$ APF. An arrow indicates strong Drl staining (magenta) in a spot colabeled with $\mathrm{mCD}$ :::GFP driven by Gal4-NP21. Gal4-NP21 labels PNs innervating DA1 robustly and innervating VA1d, v weakly at the adult stage. However, only the PNs projecting to DA1 are labeled during early pupal stages.

\section{Drl-2 mediates Wnt5 signaling}

To investigate the function of Drl-2 in Wnt5 signaling during AL development, we examined the effect of Wnt5 overexpression on glomerular organization. When Wnt5 was overexpressed in ORNs with the enhancer-trap Gal4 line, AM6, the commissure was ectopically stained with the nc82 monoclonal antibody, which labels the presynaptic protein Bruchpilot (Wagh et al., 2006) (Fig. 7B) (for AM6 expression, see supplemental Fig. 5, available at www.jneurosci.org as supplemental material). The glomerular pattern was aberrant in these flies. However, in $d r l-2$ mutants overexpressing Wnt5, ORNs did not generate the com- missural phenotype (Fig. 7C). Therefore, $d r l-2$ is epistatic to $W n t 5$ and functions downstream of Wnt5.

We performed a similar set of experiments with $d r l$ mutants. Wnt5 overexpression in ORNs of $d r l$ mutants severely affected projections; ORN axons targeted ectopic regions near the original AL positions (Fig. $7 E, E^{\prime}$ ). This enhanced phenotype is consistent with a previous report that Drl antagonizes Wnt5 signaling (Yao et al., 2007). Notably, the enhancement was ameliorated by the loss of $d r l-2$. ORN axons projected to the normal AL positions in $d r l d r l-2$ double mutants overexpressing Wnt5 (Fig. 7D). Furthermore, the glomerular pattern was similar to that of $d r l d r l-2$ 


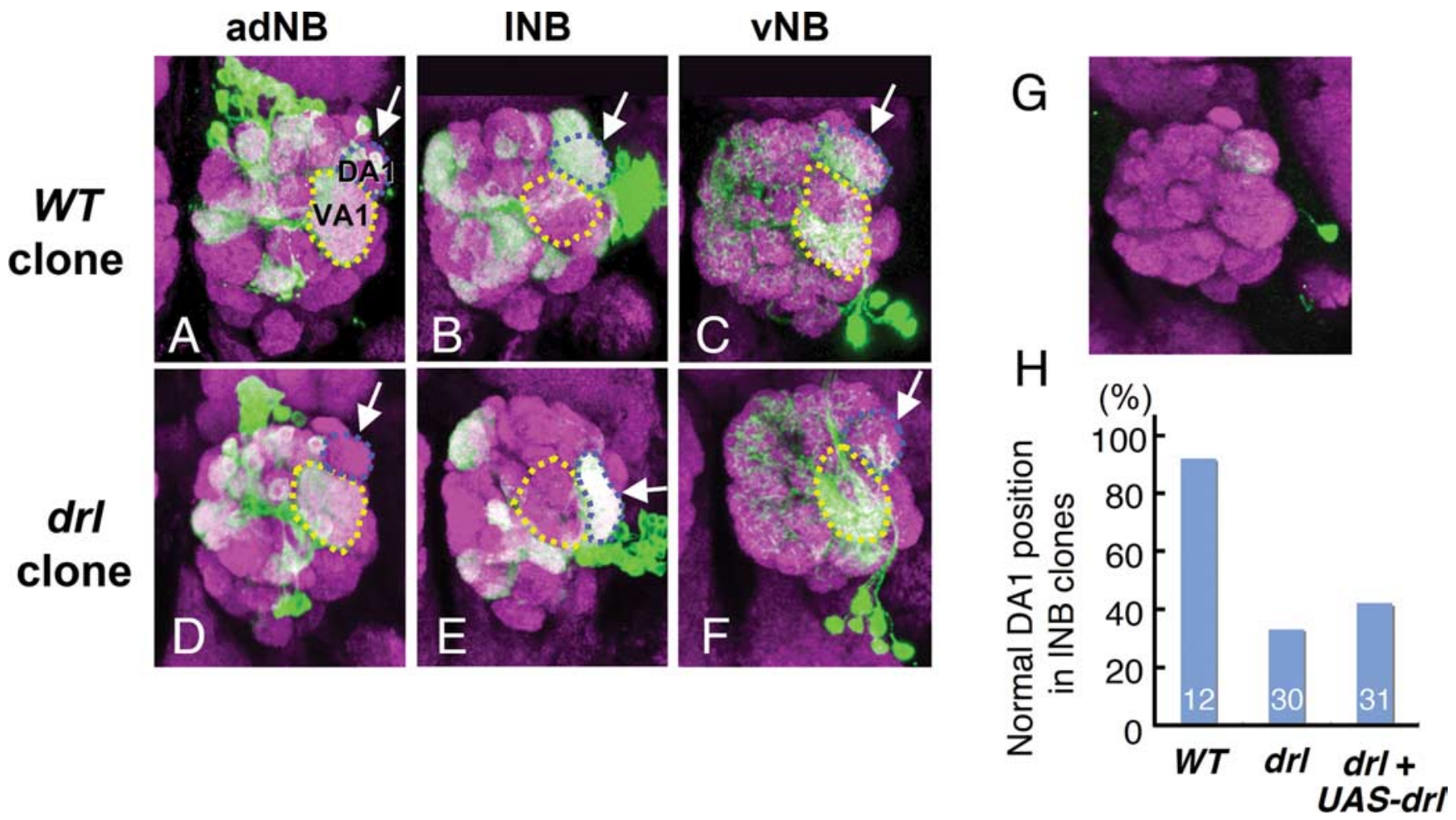

Figure 5. Glomeruli phenotypes of $d r l$ clones derived from the AL neuroblasts. $A-F$, The wild-type $(\boldsymbol{A}-\boldsymbol{C})$ and $d r l(\boldsymbol{D}-\boldsymbol{F})$ neuroblast clones are visualized by MARCM using Gal4-GH146. The clones are generated from adNB $(\boldsymbol{A}, \boldsymbol{D}), \operatorname{INB}(\boldsymbol{B}, \boldsymbol{E})$, and vNB $(\boldsymbol{C}, \boldsymbol{F})$. The blue and yellow circles represent DA1 and VA1d,v, respectively. In drl adNB clones $(\boldsymbol{D})$, the overall glomerular organization is indistinguishable from wild-type clones $(\boldsymbol{A})$, but in $\mathrm{dr} / \mathrm{INB}$ clones $(\boldsymbol{E})$, DA1 is ventrally shifted to the lateral side of VA1. In $d r / \mathrm{vNB}$ clones $(\boldsymbol{F})$, the position of DA1 is normal, but the arborization of vNB neurons into DA1 is frequently sparse or stunted. G, A single-cell clone of drl generated by MARCM using Gal4-NP21. The dendritic projection of a single drl cell to the DA1 glomerulus is normal. $\boldsymbol{H}$, The frequency at which DA1 is observed in the normal position in INB clones. The number of clones examined is shown in each bar. For details, see the text.

mutants. These findings support the hypothesis that Drl-2 is a receptor that mediates $\mathrm{Wnt} 5$ signaling.

\section{Drl-2 can substitute for Drl}

Because Drl-2 protein shares high sequence similarity with Drl, we tested whether Drl-2 can also antagonize Wnt5 signaling in a manner similar to Drl. The abnormal glomerular distribution in the $d r l$ mutant is mostly rescued by glial expression of either full-length Drl or a truncated Drl lacking the cytoplasmic domain (Yao et al., 2007). Thus, Drl may function as a decoy receptor that sequesters the Wnt5 ligand on glia. To test the effect of glial Drl-2 expression on glomerular distribution, we labeled two glomeruli, DM5 and VM2, with a specific marker line (Fig. 7F). The overall glomerular pattern was aberrant in the $d r l$ mutants and the two glomeruli were located in abnormal positions in $>60 \%$ of the ALs examined (Fig. 7G,I). When UAS-drl-2 was expressed in the glia of $d r l$ mutants under the control of Repo-Gal4, the two glomeruli were found in their normal positions in $>80 \%$ of ALs and the overall glomerular pattern was restored (Fig. $7 \mathrm{H}, I$ ). These results indicate that Drl-2 can substitute for Drl as a Wnt5 antagonist. Therefore, Drl-2 and Drl carry out similar molecular functions and Drl-2 can play opposing roles in Wnt5 signaling, depending on the cells in which it is expressed.

\section{Discussion}

In the Drosophila olfactory system, Wnt5 initiates a signaling cascade that controls the neural circuit formation including glomeruli patterning. Although Drl is known to mediate Wnt5 signaling as a receptor in the embryonic CNS (Yoshikawa et al., 2003), it exhibits an antagonistic function during olfactory system development (Yao et al., 2007). To reveal how Wnt5 signal- ing organizes the olfactory system development, we need analyze a receptor that mediates the signaling. In this study, we have identified Drl-2 as a Wnt5 receptor that is present in the olfactory system. In addition, our data suggest that Drl localized to developing glomeruli functions as a Wnt5 receptor.

Several lines of evidence support a role for Drl-2 in Wnt5 signaling. First, in $d r l-2$ mutants, two glomeruli shifted to abnormal positions as observed in Wnt5 mutants. Second, when $d r l-2$ was combined with $d r l$, the phenotype more closely resembled that of the Wnt5 mutant. Third, the abnormal commissural distribution of the presynaptic protein Bruchpilot, which was induced by overexpression of Wnt5 in ORNs, was restored in $d r l-2$ mutants. Moreover, the ectopic projection pattern of ORNs, which was caused by Wnt5 overexpression in $d r l$ mutants, was mostly ameliorated by the absence of $d r l-2$. Thus, $d r l-2$ is epistatic to both Wnt5 and $d r l$, and Drl-2 mediates Wnt5 signaling. This Drl-2 activity is antagonized by Drl during development of the olfactory system (Fig. $7 \mathrm{~J}$ ). However, the glomerular defects of $d r l$ drl-2 mutants are milder than those of Wnt5 mutants. Additional Wnt5 receptors that have yet to be identified may contribute to these phenotype differences.

Antibody staining revealed that the expression patterns of Drl and Drl-2 differ in the developing olfactory system. Drl is expressed by glia (Yao et al., 2007) and brain cells extending neurites to the AL but is not detected on ORN axons. In contrast, Drl-2 is predominantly detected on ORN axons as well as in a region adjacent to the exit site of ORN axons on the dorsal side of the AL. Thus, the expression patterns of Drl and Drl-2 are complex and mostly nonoverlapping, suggesting that multiple processes use Wnt5 signaling during olfactory system development. 

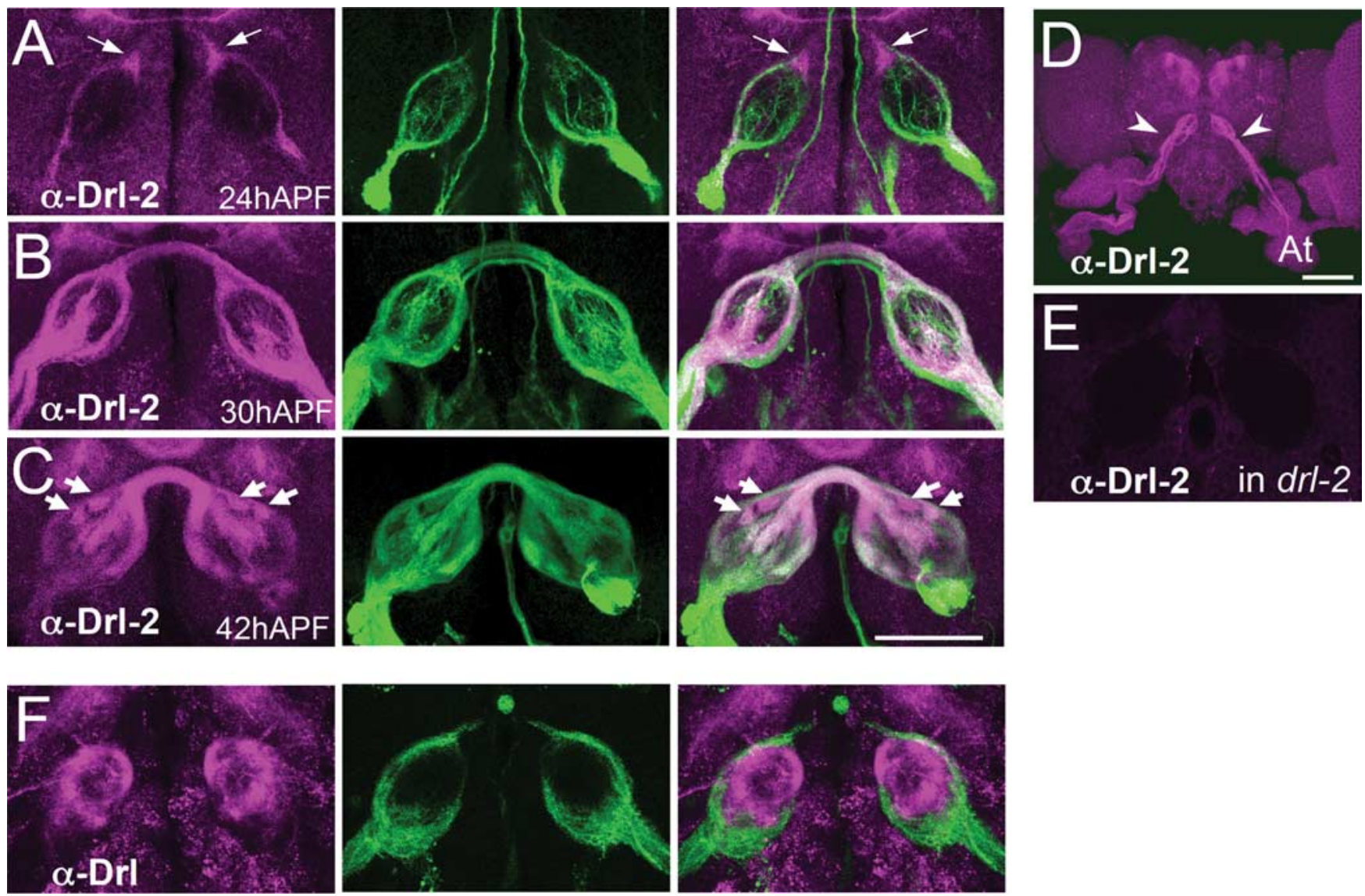

Figure 6. Developmental profile of Drl-2 expression in the AL. $\boldsymbol{A}-\boldsymbol{D}$, Immunostaining for Drl-2 (magenta) in the pupae at $24 \mathrm{~h} \mathrm{APF} \mathrm{(A),} 30 \mathrm{~h}$ APF (B, D), and $42 \mathrm{~h}$ APF (C). ORN axons are labeled with $\mathrm{mCD8}$ ::GFP (green) driven by pebbled-Gal4. The thin arrows in $A$ indicate staining in the region adjacent to the projection of ORN axons toward the commissure. In $C$, the thick arrows point to glomerular structures. Scale bar, $50 \mu \mathrm{m}$. In D, the arrowheads indicate ORN axon bundles extending from the developing antenna (At). Scale bar, $100 \mu \mathrm{m}$. $\boldsymbol{E}$, Immunostaining for Drl-2 in drl-2 mutant at $42 \mathrm{~h} \mathrm{APF.} \mathrm{F,} \mathrm{Immunostaining} \mathrm{for} \mathrm{Drl} \mathrm{(magenta)} \mathrm{at} 26 \mathrm{~h}$ APF. ORN axons are labeled with mCD8::GFP (green) driven by Gal4-SG18.1.

To reveal whether Drl-2 functions in ORNs, we performed clonal analyses in which we generated $d r l-2$ clones in ORNs. We also conducted rescue experiments by expressing Drl-2 in the ORNs of $d r l-2$ mutants with pebbled-Gal4 (data not shown). However, the mild $d r l-2$ phenotype did not allow us to clearly determine the site(s) where Drl-2 functions.

Our genetic analysis suggests that Drl and Drl-2 can both play opposing roles in Wnt5 signaling during olfactory system development. The DA1 glomerulus in $d r l$ mutant $\mathrm{lNB}$ clones shifts in a pattern similar to that of Wnt5 mutants, suggesting that Drl may transduce Wnt5 signaling in the developing AL. In addition to this possible transducing activity of Drl, the antagonistic action of Drl and the transducing activity of Drl-2 can explain other mutant glomerular phenotypes observed in this study. In $d r l$ mutant brains, in which both transducing and antagonistic activities of Drl are lost, an excess amount of Wnt5 ligand signals through Drl-2 and causes several glomeruli to shift in the direction opposite to that in Wnt5 mutants (Fig. 2D). However, in $d r l d r l-2$ mutants, in which transducing activities of both Drl and Drl-2 are lost, the glomerular defects resemble those of Wnt5 mutants (Fig. $2 F$ ). Thus, the opposing actions of Drl may both be essential for olfactory system development. In addition, we have demonstrated that Drl-2 can antagonize Wnt5 signaling when ectopically expressed in glia. Therefore, Drl and Drl-2 can each potentially mediate or antagonize Wnt5 signaling, depending on the cells in which they are expressed.

Wnt-Ryk signaling mediates both repulsion of developing ax- ons (Yoshikawa et al., 2003; Liu et al., 2005; Keeble et al., 2006; Schmitt et al., 2006) and induction of neurite growth (Lu et al., 2004; Grillenzoni et al., 2007) in Drosophila and vertebrates. In the Drosophila olfactory system, Drl localized to the neurites of AL neurons may mediate both of these activities, because $d r l \mathrm{NB}$ clones exhibit two distinct phenotypes. Neurites projecting to VAl were stunted in $d r l \mathrm{vNB}$ clones, suggesting that Drl may mediate neurite growth. In $d r l$ lNB clones, the position of DA1 was shifted ventrally, which may reflect the loss of neurite repulsion triggered by Wnt5 originating from growing ORN axons. Drl-2 on ORN axons can also mediate either of these two neurite activities to control ORN projections to the AL. Thus, proper AL development may be regulated by Wnt-Ryk signaling that mediates both the repulsion and outgrowth of neurites innervating the AL. The development of the Drosophila olfactory system appears to be controlled by a complex network of Wnt5 signaling among ORNs, interneurons, and glia.

In summary, although each of the $d r l$ and $d r l-2$ genes has acquired a specific expression pattern during evolution, both products can either mediate or antagonize Wnt5 signaling in a cell-type specific manner. In this manner, Drl and Drl-2 may regulate either the repulsion or outgrowth of neurites, perhaps in accordance with additional mechanisms. Additional studies will reveal the details of the complex Wnt5 signaling that control the formation of an accurate glomerular map of the olfactory circuitry. 

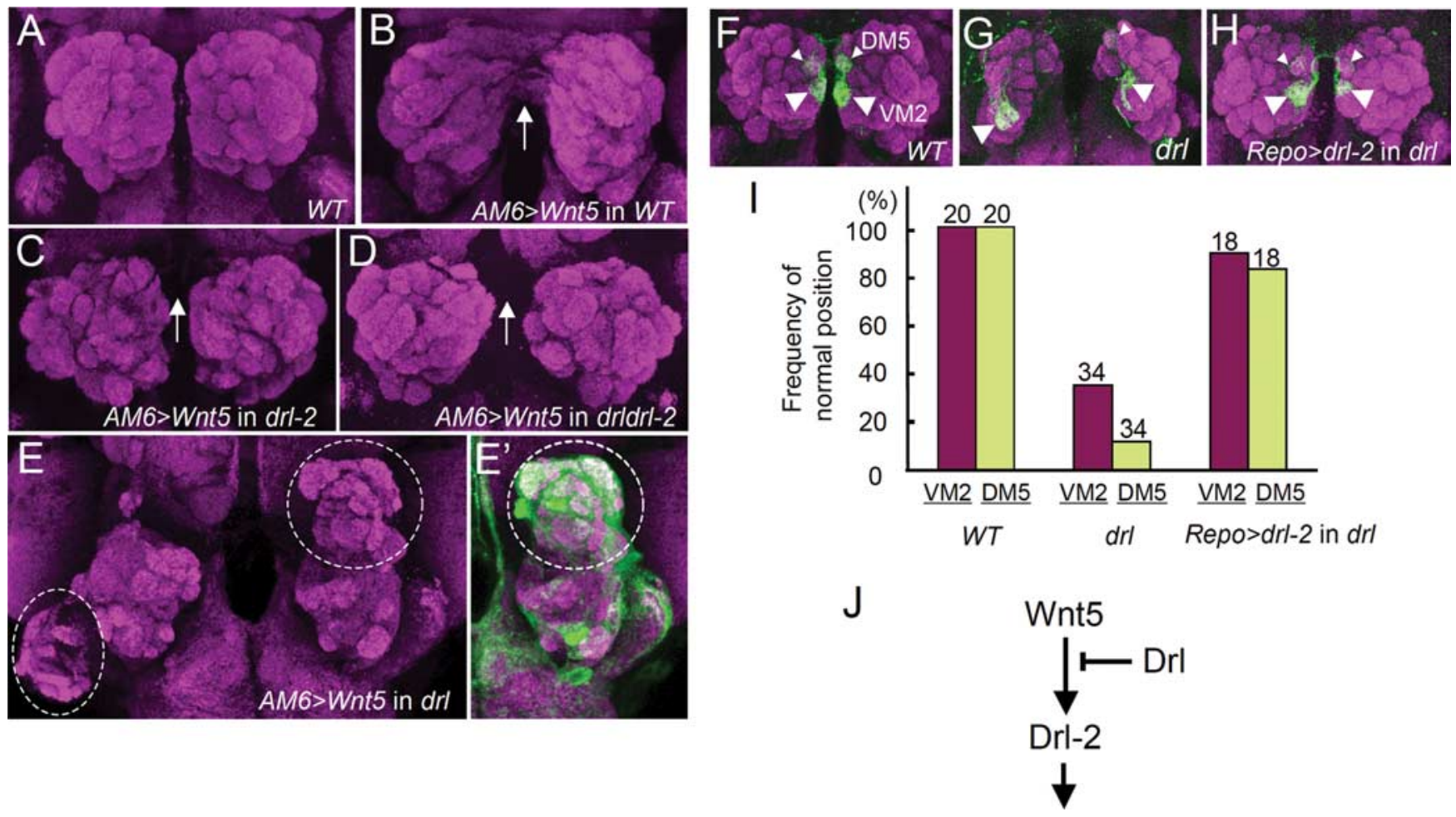

Figure 7. Drl-2 can play dual roles in Wnt5 signaling. $\boldsymbol{A}-\boldsymbol{E}^{\prime}$, Defects in the ALs. $\boldsymbol{A}$, Wild type. $\boldsymbol{B}-\boldsymbol{E}^{\prime}$, Wnt5 is overexpressed in $0 \mathrm{RN}$ s by $A M 6$ in wild type $(\boldsymbol{B}), \operatorname{drl}-2(\boldsymbol{C}), d r l d r l-2(\boldsymbol{D})$, and $d r l\left(\boldsymbol{E}, \boldsymbol{E}^{\prime}\right)$. ALs are stained with nc82 (magenta). Note that the commissure (arrow) is ectopically stained with nc 82 in $\boldsymbol{B}$, but not in $\boldsymbol{C}$ and $\boldsymbol{D}$. In $\boldsymbol{E}, 0 \mathrm{RN}$ axons are mistargeted to ectopic regions (dotted circles) that are labeled with mCD8::GFP (green) driven by AM6 as shown in $\boldsymbol{E}^{\prime}$. $\boldsymbol{F}$-I, Rescue of the drl phenotype by glial expression of Drl-2. Two glomeruli, VM2 (large arrowhead) and DM5 (small arrowhead), are labeled with Or43b-mCD8::GFP (green). $\boldsymbol{F}$, Wild type. $\mathbf{G}, \mathrm{dr} /$ mutant. $\boldsymbol{H}, \mathrm{dr} /$ mutant expressing Drl-2 in glia with Repo-Gal4. $\boldsymbol{I}$, The frequency at which VM2 and DM5 were observed in the normal position. The numbers of glomeruli examined are shown on the top of each bar.J, Regulatory circuit of Wnt5 signaling. Drl antagonizes Drl-2, which mediates Wnt5 signaling.

\section{References}

Bonkowsky JL, Yoshikawa S, O’Keefe DD, Scully AL, Thomas JB (1999) Axon routing across the midline controlled by the Drosophila Derailed receptor. Nature 402:540-544.

Callahan CA, Muralidhar MG, Lundgren SE, Scully AL, Thomas JB (1995) Control of neuronal pathway selection by a Drosophila receptor proteintyrosine kinase family member. Nature 376:171-174.

Couto A, Alenius M, Dickson BJ (2005) Molecular, anatomical, and functional organization of the Drosophila olfactory system. Curr Biol 15:1535-1547.

Endo K, Aoki T, Yoda Y, Kimura K, Hama C (2007) Notch signal organizes the Drosophila olfactory circuitry by diversifying the sensory neuronal lineages. Nat Neurosci 10:153-160.

Fishilevich E, Vosshall LB (2005) Genetic and functional subdivision of the Drosophila antennal lobe. Curr Biol 15:1548-1553.

Fradkin LG, Noordermeer JN, Nusse R (1995) The Drosophila Wnt protein DWnt-3 is a secreted glycoprotein localized on the axon tracts of the embryonic CNS. Dev Biol 168:202-213.

Grillenzoni N, Flandre A, Lasbleiz C, Dura JM (2007) Respective roles of the DRL receptor and its ligand WNT5 in Drosophila mushroom body development. Development 134:3089-3097.

Inaki M, Yoshikawa S, Thomas JB, Aburatani H, Nose A (2007) Wnt4 is a local repulsive cue that determines synaptic target specificity. Curr Biol 17:1574-1579.

Jefferis GS, Vyas RM, Berdnik D, Ramaekers A, Stocker RF, Tanaka NK, Ito K, Luo L (2004) Developmental origin of wiring specificity in the olfactory system of Drosophila. Development 131:117-130.

Jhaveri D, Rodrigues V (2002) Sensory neurons of the Atonal lineage pioneer the formation of glomeruli within the adult Drosophila olfactory lobe. Development 129:1251-1260.

Jhaveri D, Saharan S, Sen A, Rodrigues V (2004) Positioning sensory terminals in the olfactory lobe of Drosophila by Robo signaling. Development 131:1903-1912.

Keeble TR, Halford MM, Seaman C, Kee N, Macheda M, Anderson RB, Stacker SA, Cooper HM (2006) The Wnt receptor Ryk is required for
Wnt5a-mediated axon guidance on the contralateral side of the corpus callosum. J Neurosci 26:5840-5848.

Kimura K, Ote M, Tazawa T, Yamamoto D (2005) Fruitless specifies sexually dimorphic neural circuitry in the Drosophila brain. Nature 438:229-233.

Komiyama T, Johnson WA, Luo L, Jefferis GS (2003) From lineage to wiring specificity. POU domain transcription factors control precise connections of Drosophila olfactory projection neurons. Cell 112:157-167.

Komiyama T, Carlson JR, Luo L (2004) Olfactory receptor neuron axon targeting: intrinsic transcriptional control and hierarchical interactions. Nat Neurosci 7:819-825.

Komiyama T, Sweeney LB, Schuldiner O, Garcia KC, Luo L (2007) Graded expression of semaphorin-1a cell-autonomously directs dendritic targeting of olfactory projection neurons. Cell 128:399-410.

Lattemann M, Zierau A, Schulte C, Seidl S, Kuhlmann B, Hummel T (2007) Semaphorin-1a controls receptor neuron-specific axonal convergence in the primary olfactory center of Drosophila. Neuron 53:169-184.

Lee T, Luo L (1999) Mosaic analysis with a repressible cell marker for studies of gene function in neuronal morphogenesis. Neuron 22:451-461.

Liu Y, Shi J, Lu CC, Wang ZB, Lyuksyutova AI, Song XJ, Song X, Zou Y (2005) Ryk-mediated Wnt repulsion regulates posterior-directed growth of corticospinal tract. Nat Neurosci 8:1151-1159.

Lu W, Yamamoto V, Ortega B, Baltimore D (2004) Mammalian Ryk is a Wnt coreceptor required for stimulation of neurite outgrowth. Cell 119:97-108.

Luo L, Flanagan JG (2007) Development of continuous and discrete neural maps. Neuron 56:284-300.

Schmitt AM, Shi J, Wolf AM, Lu CC, King LA, Zou Y (2006) Wnt-Ryk signalling mediates medial-lateral retinotectal topographic mapping. Nature 439:31-37.

Scott K, Brady R Jr, Cravchik A, Morozov P, Rzhetsky A, Zuker C, Axel R (2001) A chemosensory gene family encoding candidate gustatory and olfactory receptors in Drosophila. Cell 104:661-673.

Stocker RF (1994) The organization of the chemosensory system in Drosophila melanogaster: a review. Cell Tissue Res 275:3-26. 
Stocker RF, Heimbeck G, Gendre N, de Belle JS (1997) Neuroblast ablation in Drosophila P[GAL4] lines reveals origins of olfactory interneurons. J Neurobiol 32:443-456.

Sweeney LB, Couto A, Chou YH, Berdnik D, Dickson BJ, Luo L, Komiyama T (2007) Temporal target restriction of olfactory receptor neurons by Semaphorin-1a/PlexinA-mediated axon-axon interactions. Neuron 53:185-200.

Tanaka NK, Awasaki T, Shimada T, Ito K (2004) Integration of chemosensory pathways in the Drosophila second-order olfactory centers. Curr Biol 14:449-457.

Toba G, Ohsako T, Miyata N, Ohtsuka T, Seong KH, Aigaki T (1999) The gene search system. A method for efficient detection and rapid molecular identification of genes in Drosophila melanogaster. Genetics 151:725-737.

Vosshall LB, Amrein H, Morozov PS, Rzhetsky A, Axel R (1999) A spatial map of olfactory receptor expression in the Drosophila antenna. Cell 96:725-736.

Wagh DA, Rasse TM, Asan E, Hofbauer A, Schwenkert I, Dürrbeck H, Buchner S, Dabauvalle MC, Schmidt M, Qin G, Wichmann C, Kittel R, Sigrist SJ, Buchner E (2006) Bruchpilot, a protein with homology to ELKS/ CAST, is required for structural integrity and function of synaptic active zones in Drosophila. Neuron 49:833-844.

Yao Y, Wu Y, Yin C, Ozawa R, Aigaki T, Wouda RR, Noordermeer JN, Fradkin LG, Hing H (2007) Antagonistic roles of Wnt5 and the Drl receptor in patterning the Drosophila antennal lobe. Nat Neurosci 10:1423-1432.

Yoshikawa S, McKinnon RD, Kokel M, Thomas JB (2003) Wntmediated axon guidance via the Drosophila Derailed receptor. Nature 422:583-588. 